International Braz J Urol

Vol. 34 (6): 708-714, November - December, 2008

\title{
Transcutaneous Electrical Nerve Stimulation (TENS) in the Symptomatic Management of Chronic Prostatitis/Chronic Pelvic Pain Syndrome: A Placebo-Control Randomized Trial
}

\author{
Lamina Sikiru, Hanif Shmaila, Samani A. Muhammed
}

Department of Physiotherapy/Physiology (LS), Faculty of Medical Sciences/Jimma Specialized Hospital, Jimma University, Jimma, Ethiopia, Department of Physiotherapy (HS), Murtala Mohammad Specialist Hospital, Kano, Nigeria, Department of Urology/Surgery (SAM), Murtala Mohammad Specialist Hospital, Kano, Nigeria

\begin{abstract}
Objective: The aim of the study was to investigate the therapeutic efficacy of transcutaneous electrical nerve stimulation (TENS) in the symptomatic management of chronic prostatitis pain/chronic pelvic pain syndrome.

Design: A pretest, posttest randomized double blind design was used in data collection.

Participant: Twenty-four patients diagnosed with chronic prostatitis- category IIIA and IIIB of the National Institute of Health Chronic Pain (NIH-CP) were referred for physiotherapy from the Urology department.

Intervention: Pre treatment pain level was assessed using the NIH-CP (pain domain) index. The TENS group received TENS treatment, 5 times per week for a period of 4 weeks (mean treatment frequency, intensity, pulse width and duration of $60 \mathrm{~Hz}, 100 \mu \mathrm{S}, 25 \mathrm{~mA}$ and 20 minutes respectively). The Analgesic group received no TENS treatment but continued analgesics; the Control group received no TENS and Analgesic but placebo. All subjects were placed on antibiotics throughout the treatment period.

Outcome measures: Post-treatment pain level was also assessed using NIH-CP pain index.

Result: Findings of the study revealed significant effect of TENS on chronic prostatitis pain at $\mathrm{p}<0.05$.

Conclusion: TENS is an effective means of non-invasive symptomatic management of chronic prostatitis pain.
\end{abstract}

Key words: pain; TENS; chronic prostatitis; chronic pelvic pain

Int Braz. J Urol. 2008; 34: 708-14

\section{INTRODUCTION}

In 1995 the National Institutes of Health (NIH) classified prostatitis into 4 main categories: 1) acute bacterial; 2) chronic bacterial; 3) non-bacteria chronic prostatitis/chronic pelvic pain syndrome $(\mathrm{CP} /$ CPPS); 4) asymptomatic inflammatory. The CP/CPPS was further subdivided into inflammatory (category IIIA) and non-inflammatory (category IIIB) prostatitis (1).
Chronic prostatitis/chronic pelvic pain syndrome (CP/CPPS), the subject of the present study is a debilitating condition diagnosed in the presence of chronic pelvic pain and lower urinary tract symptoms (2). CP/CPPS is the most common (3), yet most poorly understood "prostatitis syndrome" (4).CP/CPPS is truly a devastating disease.

A new perception of CP/CPPS appeared following the $1995 \mathrm{NIH} / \mathrm{NIDDK}$ workshop, which emphasized the importance of pain as the hallmark 
of $\mathrm{CP} / \mathrm{CPPS}$ and questioned the role of the prostate in producing the symptoms (2).

Chronic pelvic pain syndrome (NIH category III) and commonly manifests as pain in areas including the perineum, rectum, prostate, penis, testicles, and abdomen (5). The use of antibiotics in NIH category III is based on the uncertain etiology and the possibility that a potential pathogen or a cryptic non-culturable organism may be causative (6). Combination of analgesics, alpha-blockers (tamsulosin) antibiotics (TMP-SMX, fluoroquinolones or tetracycline), and muscle relaxants such as diazepam coupled with prostatic massage and supportive therapy (perineal support, pelvic floor physiotherapy, biofeedback and relaxation therapy) has been reported to yield higher cure rate and relief of pain and voiding symptoms compared to antibiotics alone and is the treatment option favored by most urologists (7).

However, no highly effective therapy has been identified. Thus far, strategies have focused on symptomatic relief (8). In addition, it is not clear whether therapy for IIIA and IIIB prostatitis syndromes should differ because the role of inflammation in these syndromes is incompletely understood (6).

Transcutaneous electrical nerve stimulation (TENS) was introduced as an alternatively therapy to pharmacological treatments for chronic pain. TENS currently is one of the most commonly used forms of electro analgesia. Hundreds of clinical reports exist concerning the use of TENS for various types of conditions such as low back pain, myofascial and arthritic pain, sympathetically mediated pain, neurogenic pain, visceral pain, and post-surgical pain (9-12).

The widespread use of TENS is useful for a wide range of chronic pain conditions $(10,11)$. TENS is the application of pulsed square wave current through surface electrodes placed on the skin, to the peripheral nerve fibers for the control of pain (13). TENS is a non-invasive and non-addictive treatment (13). TENS does not produce anesthesia or nerve block (14).

Small uncontrolled studies have shown limited improvements in scores on the NIH Chronic Prostatitis Symptom Index with the use of biofeedback $(15,16)$ and acupuncture (17). Physical therapies, including prostatic massage and sitz baths, have been recommended but have not been adequately studied.
The needs for the symptomatic management of pain in CP/CPPS with a non-invasive, non pharmacological, non-addictive technique such as TENS clearly exist. The purpose of the present study was therefore to determine the efficacy of TENS in the symptomatic management of $\mathrm{CP} / \mathrm{CPPS}$.

\section{MATERIALS AND METHODS}

Design - In this study, a double blind randomized pre-test, post-test independent placebo-control design was used.

Participants - The participants for this study included 24 diagnosed CP/CPPS patients attending the Urology Department of Murtala Mohammad Specialist Hospital (MMSH) and from private urologists. The inclusion criteria were randomly selected men between 24-50 years, previously diagnosed as category IIIA or IIIB CP/CPPS. Exclusion criteria were prostate and other urogenital cancer and infection, loss of skin sensation at and around painful area, cardiac pace maker, previous exposure to TENS and other electro analgesia.

Instrumentation -

1. TENS generated from ENS 931 (Enraf Nonius), Holland, with two conducting rubber electrodes and moist pads (size $3 \mathrm{~cm} \mathrm{X} 6 \mathrm{~cm}$ ).

2. TENS gel (Aquasonic gel) (J.J. Industry, Seoul, Korea).

3. NIH chronic prostatitis symptom index (NIHCPSI) pain domain questionnaire.

Intervention - Those not on analgesic for at least one week and had not received any form of electromagnetic/acupuncture or heat therapy were recruited for the study. Informed consent was sought from subjects willing to participate in accordance with the ethics of human participation by the Ethical Committee of Murtala Mohammad Specialist Hospital, Kano. Pre treatment pain assessment was conducted by a neutral Assessor (Physiotherapist). NIH chronic prostatitis symptom index questionnaire, the pain domain describing the location, frequency and severity of pain was presented to each patient and instruction was given to indicate the pain characteristics and level by signifying a number on the scale. Subjects were then randomly assigned into three groups: 
- $\mathrm{X}_{1}$ (TENS group): Antibiotics + TENS only (n $=8)$

- $\mathrm{X}_{2}$ (Analgesic group): Antibiotics + Analgesic only $(\mathrm{n}=8)$

- $\mathrm{X}_{3}$ (Control group): Antibiotics only + placebo tablets $(\mathrm{n}=8)$

Patients in the TENS group continued their antibiotics (ofloxacin) as prescribed by their Physician. The rationale for ofloxacin (300 $\mathrm{mg}$ t.d.s.) usage was because it is considered the recommended drug for chronic nonbacterial prostatitis management, covering culture-negative germs like Chlamydia (3).

For TENS application, patients were comfortably positioned based on the painful area (to cover the perineal-suprapubic region) for electrode placement. Sensory test was conducted on the skin over the painful area by using two test tubes with cold and warm water, also light touch via pin prick. It was ascertained that sensitivity of the area was intact, and that there was no resistance, this allowed for effective stimulation. TENS gel was applied on the surface of the electrodes to aid maximum transmission of current. Electrodes were placed on the skin overlying the painful area and held firmly in position as described by Radhakrishnan and Sluka (18); Oosterhof et al. (19).

The machine was switched on; a suitable and comfortable frequency and pulse width were selected on the stimulator by turning the appropriate knobs. Intensity knob was turned to a level when the patients felt a tingling or pins and needle sensation, the intensity was then reduced to a level that the patient reported a comfortable stimulation. Painful TENS was avoided.

Patients were stimulated with high TENS daily for an average of 20 minutes, mean frequency, pulse width and intensity of $100 \mathrm{~Hz}, 100 \mu$ s and $25 \mathrm{~mA}$ respectively for a mean duration daily, 5 times per week for 4 consecutive weeks (average of 20 treatment sessions) (18-21).

The analgesic group continued with their antibiotics and analgesics (ibuprofen $400 \mathrm{mg}$ b.d.); while the control group continued with their antibiotics (ofloxacin) and placebo tablets as prescribed by their physician for the same period. Seven days prior to their next medical consultation, after patients felt that they had exhausted their analgesic and TENS treatment was stopped ( 7 days post treatment [wash out period]).

Outcome measures - All subjects were assessed for the Post-treatment pain score using the same pre treatment procedure by the same neutral assessor who had no prior knowledge of the study, subjects' records or groups.

Data analysis - Mean and standard deviation (SD) were computed. Kruskal Wallis test and post hoc group differences were computed for the pre- and post-treatment pain values. Statistical analysis was performed on microcomputer using Statistical Package for the Social Sciences - SPSS (Windows Version 15.0, Chicago, IL.) A probability level of 0.05 or less was used to indicate statistical significance.

\section{RESULTS}

The age of subjects ranged from 24 to 50 years mean $\pm \mathrm{SD}(38.17 \pm 8.75), 23$ to 55 years $(45.38 \pm$ $11.16)$ and 30 to 60 years $(46.83 \pm 8.16)$ for TENS $\left(\mathrm{X}_{1}\right)$, Analgesic $\left(\mathrm{X}_{2}\right)$ and Control $\left(\mathrm{X}_{3}\right)$ groups respectively.

The result of the present study indicated significant effect of TENS on chronic prostatitis pain. Table-1 shows the group mean and SD of pre

Table 1 - Groups mean, SD and mean rank pre-test and post-test pain values $(n=24)$.

\begin{tabular}{lccccc}
\hline & & Pre-test & \multicolumn{2}{c}{ Post-test } & \\
Variables & N & Mean \pm SD & Mean rank & Mean \pm SD & Mean Rank \\
\hline TENS group pain & 8 & $16.38 \pm 2.88$ & 10.00 & $9.00 \pm 0.93$ & 4.50 \\
Analgesic group pain & 8 & $17.13 \pm 4.91$ & 11.13 & $13.38 \pm 1.50$ & 13.38 \\
Control group pain & 8 & $20.25 \pm 3.73$ & 16.38 & $15.88 \pm 1.55$ & 19.63 \\
\hline
\end{tabular}


Table 2 - Kruskal Wallis summary for groups' pain level.

\begin{tabular}{lcccccc}
\hline Variables & N & Mean & SD & df & Chi-square & p Value \\
\hline Pre-test pain & 24 & 7.92 & 4.13 & 2 & 3.752 & 0.153 \\
Post-test pain & 24 & 2.75 & 3.17 & 2 & 18.804 & $0.000^{*}$ \\
\hline
\end{tabular}

$X_{(2.24)} ; p<0.05 ; *$ significant; $S D=$ standard deviation.

and post-test pain values (levels). Table-2 shows the pretest-post-test mean, standard deviation and Kruskal Wallis analysis. Groups pain level did not differ significantly in the pretest pain values $\left(\mathrm{X}^{2}=3.752 \mathrm{p}=\right.$ 0.153 ), while the post-test pain values differ significantly $\left(\mathrm{X}^{2}=18.804, \mathrm{p}=0.000\right)$.

Table-3 further showed a significant effect of TENS group over other groups at $p<0.05$. Post hoc analysis indicated significant effect of TENS over analgesic $(1 \& 2[\mathrm{~K}=3.105])$, placebo $(1 \& 3[\mathrm{~K}=$ 5.315]). Analgesic and placebo did not differ significantly $(2 \& 3[\mathrm{~K}=2.1746])$.

\section{COMMENTS}

The purpose of the present study was to investigate the therapeutic efficacy of TENS in the symptomatic management of chronic pain in CP/CPPS. The result showed an appreciable effect of TENS in the symptomatic management of chronic pain in $\mathrm{CP} / \mathrm{CPPS}$. The predominant symptom of $\mathrm{CP} / \mathrm{CPPS}$ is pain. Therefore, modalities to treat pain specifically may be effective. There is mounting evidence that the pain of CP/CPPS may be neuropathic and associated with central nervous system changes. The presence of central sensitization in patients with $\mathrm{CP} / \mathrm{CPPS}$ was demonstrated by Yang and colleagues (22), who compared thermal algometry in men with $\mathrm{CP} / \mathrm{CPPS}$ versus asymptomatic controls. Men with $\mathrm{CP} / \mathrm{CPPS}$ reported a higher visual analog scale to short bursts of noxious heat stimuli to the perineum but no difference to the anterior thigh. Thus, these patients have altered sensation in the perineum compared with controls.

Many studies have investigated the effects of complementary and alternative medicine (CAM) strategies in the management of CP/CPPS. The result of the present study was in agreement with a similar non invasive CAM therapy, reported by Capidice et al. (23). In their pilot study, they investigated the effect of acupuncture in 10 men diagnosed as CP/CPPS (category IIIA or IIIB). Acupuncture was applied for 30 minutes, twice weekly for 6 weeks. They reported significant decrease in NIH-CPSI for pain and lower urinary tract symptoms and quality of life.

Another similar study was conducted by John and co-workers (24). Their study tested a high frequency, urethral-anal prototype stimulation device in men with CP/CPPS twice weekly for 5 weeks. The results demonstrated a significant decrease in the NIHCPSI $(\mathrm{P}=0.0002)$ with no urethral, anal complaints or other side effects The authors suggest that due to the positive results, simple technology and ability to be self-administered, this new device may be useful in the treatment of CP/CPPS.

Two similar studies $(25,26)$ on non pharmacological, non invasive CAM therapy testing the value of biofeedback therapy for CP/CPPS yielded

Table 3 - Post hock paired comparisons.

\begin{tabular}{lccc}
\hline & K1 & K2 & K3 \\
\hline Post pain KI & - & $3.105^{*}$ & $5.315^{*}$ \\
Post pain K2 & $3.105^{*}$ & - & 2.175 \\
Pre pain K3 & $5.315^{*}$ & 2.175 & - \\
\hline
\end{tabular}

$F($ table value $)=2.89 ; p<0.05 ; *$ significant . 
positive results. The first study assessed 62 patients who were refractory to conventional therapy (such as antibiotics and/or alpha-blockers) for greater than half a year. These patients were treated utilizing the Urostym Biofeedback equipment five times a week for 2 weeks with a stimulus intensity of $15-23 \mathrm{~mA}$ and duration of $20 \mathrm{~min}$. The NIH-CPSI index noted a significant overall reduction in score $(\mathrm{P}<0.01)$ and no side effects were reported during the trial (25).

A second pilot study evaluated biofeedback therapy in 19 men with pelvic floor tension and $\mathrm{CP} / \mathrm{CPPS}$. These results demonstrated significant improvement in pain scores as measured by the AUA symptom index $(P=0.001)$. While this study focused on testing the effect of biofeedback therapy in treating the symptoms associated with CP/CPPS, it also implicated the presence of pelvic floor tension contributing to pain and the paramount importance of muscular reeducation for its treatment (26). These initial, positive biofeedback studies may warrant larger randomized clinical trials to confirm safety and efficacy as well as explore the mechanism of action of biofeedback therapy.

Many studies $(9,11,12,27,28)$ have reported significant effect of TENS on visceral pain such as labor pain and dysmenorrhea. Based on this, TENS may be indicated in the management of chronic prostatitis pain; a similar visceral organ. Although there is no better way of eliminating pain than by removing its cause. With any symptomatic therapy, however, efficacy must be weighed with the risks involved. TENS might be preferable to large amount of analgesics and their side effects. Also, TENS is readily available to both patients and therapists, cheaper and easy to apply compared to other non invasive, non pharmacological complementary and alternative medicine therapies. Based on the result of the present study, the authors hereby concluded that TENS is an effective means of non-invasive, non pharmacological symptomatic management of chronic prostatitis pain.

Though, the present study indicated significant efficacy of TENS on chronic pain in CP/CPPS. However, there are some limitations of the study; they included the non availability of data on long term efficacy of TENS, few numbers of participants, non sham TENS group and failure to distinguished treatment between CP/CPPS category IIIA and IIIB.
These limiting factors warrant more attention in future studies before a conclusive statement could be made. However, the present study could provide the relevant data in which future studies could base on.

\section{ACKNOWLEDGMENT}

The authors acknowledge the staff of the Department of Physiotherapy, Murtala Mohammed Specialist Hospital.

\section{CONFLICT OF INTEREST}

\author{
None declared.
}

\section{REFERENCES}

1. Bartoletti R, Mondaini N, Pavone C, Dinelli N, Prezioso D: Introduction to chronic prostatitis and chronic pelvic pain syndrome (CP/CPPS). Arch Ital Urol Androl. 2007; 79: 55-7.

2. Collins MM, Stafford RS, O'Leary MP, Barry MJ: How common is prostatitis? A national survey of physician visits. J Urol. 1998; 159: 1224-8.

3. Calhoun EA, McNaughton Collins M, Pontari MA, O'Leary M, Leiby BE, Landis RJ, et al.: The economic impact of chronic prostatitis. Arch Intern Med. 2004; 164: 1231-6.

4. Krieger JN, Nyberg L Jr, Nickel JC: NIH consensus definition and classification of prostatitis. JAMA. 1999; 282: 236-7.

5. Litwin MS, McNaughton-Collins M, Fowler FJ Jr, Nickel JC, Calhoun EA, Pontari MA, et al.: The National Institutes of Health chronic prostatitis symptom index: development and validation of a new outcome measure. Chronic Prostatitis Collaborative Research Network. J Urol. 1999; 162: 369-75.

6. Gurunadha Rao Tunuguntla HS, Evans CP: Management of prostatitis. Prostate Cancer Prostatic Dis. 2002; 5: 172-9.

7. Barbalias GA, Nikiforidis G, Liatsikos EN: Alphablockers for the treatment of chronic prostatitis in combination with antibiotics. J Urol. 1998; 159: 883-7.

8. Schaeffer AJ: Clinical practice. Chronic prostatitis and the chronic pelvic pain syndrome. N Engl J Med. 2006; 355: 1690-8. 
9. Chao AS, Chao A, Wang TH, Chang YC, Peng HH, Chang SD, et al.: Pain relief by applying transcutaneous electrical nerve stimulation (TENS) on acupuncture points during the first stage of labor: a randomized double-blind placebo-controlled trial. Pain. 2007; 127: 214-20.

10. Carroll D, Moore RA, McQuay HJ, Fairman F, Tramèr M, Leijon G: Transcutaneous electrical nerve stimulation (TENS) for chronic pain. Cochrane Database Syst Rev. 2001; (3): CD003222.

11. Ying KN, While A: Pain relief in osteoarthritis and rheumatoid arthritis: TENS. Br J Community Nurs. 2007; 12: 364-71.

12. Akinbo SR, Onwudimegwu WN, Ajayi GO: Evaluation of the efficacy of TENS compared with analgesics in the management of primary dysmenorrhoea. Journal of Nigeria Medical Rehabilitation Therapists. 2000; 5: 27-30.

13. Akinbo SRA, Oyedele SY, Shaba OP: Transcutaneous electrical nerve stimulation (TENS) in the management of temporomandibular joint pain and dysfunction syndrome. Journal of The Nigeria Medical Rehabilitation Therapists 2003; 8: 32-5.

14. Wall PD: The discovery of TENS. Physiotherapy. 1985; 71: 348-50.

15. Anderson RU, Wise D, Sawyer T, Chan C: Integration of myofascial trigger point release and paradoxical relaxation training treatment of chronic pelvic pain in men. J Urol. 2005; 174: 155-60.

16. Nadler RB: Bladder training biofeedback and pelvic floor myalgia. Urology. 2002; 60(Suppl 6): 42-3; discussion 44.

17. Chen R, Nickel JC: Acupuncture ameliorates symptoms in men with chronic prostatitis/chronic pelvic pain syndrome. Urology. 2003; 61: 1156-9; discussion 1159.

18. Radhakrishnan R, Sluka KA: Deep tissue afferents, but not cutaneous afferents, mediate transcutaneous electrical nerve stimulation-Induced antihyperalgesia. J Pain. 2005; 6: 673-80.

19. Oosterhof J, De Boo TM, Oostendorp RA, WilderSmith OH, Crul BJ: Outcome of transcutaneous electrical nerve stimulation in chronic pain: short-term results of a double-blind, randomised, placebo-controlled trial. J Headache Pain. 2006; 7: 196-205.

20. Chandran P, Sluka KA: Development of opioid tolerance with repeated transcutaneous electrical nerve stimulation administration. Pain. 2003; 102: 195201.

21. Gopalkrishnan P, Sluka KA: Effect of varying frequency, intensity, and pulse duration of transcutaneous electrical nerve stimulation on primary hyperalgesia in inflamed rats. Arch Phys Med Rehabil. 2000; 81: 984-90.

22. Yang CC, Lee JC, Kromm BG, Ciol MA, Berger RE: Pain sensitization in male chronic pelvic pain syndrome: why are symptoms so difficult to treat? J Urol. 2003; 170: 823-6; discussion 826-7.

23. Capodice JL, Jin Z, Bemis DL, Samadi D, Stone BA, Kapan S, Katz AE: A pilot study on acupuncture for lower urinary tract symptoms related to chronic prostatitis/chronic pelvic pain. Chin Med. 2007; 2: 1.

24. John H, Rüedi C, Kötting S, Schmid DM, Fatzer M, Hauri D: A new high frequency electrostimulation device to treat chronic prostatitis. J Urol. 2003; 170: 1275-7.

25. Ye ZQ, Cai D, Lan RZ, Du GH, Yuan XY, Chen Z, et al.: Biofeedback therapy for chronic pelvic pain syndrome. Asian J Androl. 2003; 5: 155-8.

26. Clemens JQ, Nadler RB, Schaeffer AJ, Belani J, Albaugh J, Bushman W: Biofeedback, pelvic floor re-education, and bladder training for male chronic pelvic pain syndrome. Urology. 2000; 56: 951-5.

27. American College of Obstetrician and Gynecologists Committee on Practice Bulletins -- Gynecology. ACOG Practice Bulletin No. 51. Chronic pelvic pain. Obstet Gynecol. 2004; 103: 589-605.

28. Brosseau L, Yong K, Marchand S, Robinson V, Wells G, Tugwell P. Efficacy of TENS for rheumatoid arthritis:a systematic review. Physical Therapy Review. 2002; 7: 199-208.

\author{
Accepted after revision: \\ August 28, 2008
}




\section{EDITORIAL COMMENT}

Chronic prostatitis (CP) is one of the most prevalent conditions in urology, and represents an important international health problem. Throughout the past century, the diagnostic entity of CP has been recognized and its clinical characteristics well described. However, despite the multiple approaches to management of $\mathrm{CP}$, no hard and fast guidelines have been developed.

The new perception of $\mathrm{CP} /$ Chronic Pelvic Pain Syndrome (CPPS) following the 1995 NIH/NIDDK workshop has emphasized the importance of pain as the hallmark of CP/CPPS. The authors investigated the therapeutic efficacy of transcutaneous electrical nerve stimulation (TENS) in the symptomatic management of $\mathrm{CP} / \mathrm{CPPS}$. This placebo-control

\section{EDITORIAL COMMENT}

The authors are to be congratulated for an innovative approach to managing chronic pelvic pain syndrome in men, commonly referred to as chronic prostatitis. Multiple randomized placebo-controlled trials of oral pharmaceutical agents, including antibiotics, non-steroidal anti-inflammatory drugs, alpha blockers, and hormone blocking agents have been unsuccessful in ameliorating chronic pelvic pain symptoms. More local therapy is warranted. The need for symptomatic management of chronic prostatitis/chronic pelvic pain syndrome (CPPS) is certainly germane where no clear biological pathogenetic mechanism has been elucidated.

This approach to pain management needs verification with a sham treatment control. As with new surgical investigations that is a difficult clinical trial to devise. It is a stretch to describe this pilot trial as a double blind randomized placebo-controlled design. If we are to believe that neural dermatomes can act as pathways for counter-irritant stimulation that inhibits painful conception, then TENS is a good randomized study show significant improvement in scores on the NIH-CP pain index with the use of TENS. Based on the present study, the authors concluded that TENS is an effective means of non-invasive, non pharmacological symptomatic management of chronic prostatitis pain. However, we still need more high quality multi-center randomized controlled trials from other countries and regions.

\section{Dr. J. R. Yang Department of Urology Second Xiang-Ya Hospital Central South University Changsha 410011, China E-mail:yjinrui@yahoo.com}

alternative. The endurance of a positive response to TENS needs to be assessed considerably longer than 4 weeks. Most treatment trials in chronic pelvic pain syndromes utilize a minimum 12-week observation period to endpoint.

Fortunately TENS application lends itself to patient controlled administration and intermittent personal selection of usage frequency. This is a huge advantage. It is akin to utilizing intermittent tibial nerve electrical neuromodulation for overactive bladder symptoms. Daily stimulation may not be necessary. In general, electrical neuromodulation applications continue to suggest avenues of pursuit that should be encouraged.

Dr. Rodney U. Anderson Department of Urology Stanford University School of Medicine Stanford, California 94305, USA E-mail:rua@stanford.edu 